International Electronic Journal of Algebra

Volume 30 (2021) 99-115

DOI: $10.24330 /$ ieja.969592

\title{
SOME PROPERTIES OF STAR OPERATIONS ON RING EXTENSIONS
}

\author{
Lokendra Paudel and Simplice Tchamna
}

Received: 4 February 2020; Revised: 5 September 2020; Accepted: 29 November 2020

Communicated by Abdullah Harmancı

\begin{abstract}
Let $\star$ be a star operation on a ring extension $R \subseteq S$. A ring extension $R \subseteq S$ is called Prüfer star-multiplication extension $(\mathrm{P} \star \mathrm{ME})$ if $\left(R_{[\mathfrak{m}]}, \mathfrak{m}_{[\mathfrak{m}]}\right)$ is a Manis pair in $S$ for every $\star$-maximal ideal $\mathfrak{m}$ of $R$. We establish some results on star operations, and we study $\mathrm{P} \star \mathrm{ME}$ in pullback diagrams of type $\square$. We show that, for a maximal ideal $\mathfrak{m}$ of $R$, the extension $R_{[\mathfrak{m}]} \subseteq S$ is Manis if and only if $R[X]_{[\mathfrak{m} R[X]]} \subseteq S[X]$ is a Manis extension.
\end{abstract}

Mathematics Subject Classification (2020): 13A15, 13A18, 13B02

Keywords: Star operation, ring extension, Prüfer extension

\section{Introduction}

In this article, all rings are commutative with identity. Let $R \subseteq S$ be a ring extension. In [8, Definition 1, p. 139], M. Knebusch and T. Kaiser define a star operation on the extension $R \subseteq S$ to be a map $\star: \mathcal{J}(R, S) \longrightarrow \mathcal{J}(R, S)$, where $\mathcal{J}(R, S)$ is the set of all $R$-submodules of $S$, satisfying the following conditions for all $A, B \in \mathcal{J}(R, S)$.

(c $\left.c_{1}\right) \subseteq A^{\star}$.

$\left(c_{2}\right)$ If $A \subseteq B$, then $A^{\star} \subseteq B^{\star}$.

$\left(c_{3}\right)\left(A^{\star}\right)^{\star}=A^{\star}$.

(c $\left.c_{4}\right) A B^{\star} \subseteq(A B)^{\star}$.

A star operation $\star$ on a ring extension $R \subseteq S$ is said to be strict if $R^{\star}=R[8$, Definition 1, p. 139]. A star operation $\star$ on a ring extension $R \subseteq S$ is said to be of finite type if for each $R$-submodule $A$ of $R, A^{\star}=\bigcup K^{\star}$, where $K$ ranges over all the finitely generated $R$-submodules of $S$ contained in $A$ [8, Definition 1, p. 156] .

Remark 1.1. [8, Proposition 6.3, p.156] For a star operation $\star: \mathcal{J}(R, S) \longrightarrow$ $\mathcal{J}(R, S)$, and each $R$-submodule $A$ of $S$, define $A^{\star_{f}}=\bigcup K^{\star}$, where $K$ ranges over all the finitely generated $R$-submodules of $S$ contained in $A$. Then the map $\star_{f}: \mathcal{J}(R, S) \longrightarrow \mathcal{J}(R, S)$ defined by $A \longmapsto A^{\star_{f}}$ is a star operation of finite type on the extension $R \subseteq S$. 
Remark 1.2. Let $R \subseteq S$ be a ring extension, and $\star$ be a star operation on the extension $R \subseteq S$. If $A$ is an $R$-submodule of $S$ such $A^{\star}=R^{\star}$, then $\left(A^{n}\right)^{\star}=R^{\star}$ for each positive integer $n$. In fact, if $A^{\star}=R^{\star}$, then by $[8$, Proposition 4.1(a), p. 146] we have $\left(A^{n}\right)^{\star}=(\underbrace{A \cdots A}_{n \text { times }})^{\star}=(\underbrace{A^{\star} \cdots A^{\star}}_{n \text { times }})^{\star}=(\underbrace{R^{\star} \cdots R^{\star}}_{n \text { times }})^{\star}=R^{\star}$.

Let $I$ be an ideal of $R$. The ideal $I$ is said to be a $\star$-ideal if $I^{\star}=I$. Following the terminology use in [1], we called an ideal $I$ of $R$ a $\star$-prime ideal if $I$ is both a star-ideal and a prime ideal of $R$. A maximal element in the set of all $\star$-ideal of $R$ is called $\star$-maximal ideal.

Lemma 1.3. [12, Remark 2.4] Let $R \subseteq S$ be a ring extension, and let $\star$ be a strict star operation of finite type on $R \subseteq S$. Then each proper $\star$-ideal of $R$ is contained in $a \star$-prime ideal of $R$ (which is also $a \star$-maximal ideal of $R$ ).

Let $R \subseteq S$ be a ring extension, and let $A$ be an $R$-submodule of $S$. The $R$ submodule $A$ is said to be $S$-regular if $A S=S$ [7, Definition 1, p. 84]. The $R$-submodule $A$ of $S$ is called $S$-invertible, if there exists an $R$-submodule $B$ of $S$ such that $A B=R\left[7\right.$, Definition 3 , p. 90]. In this case, we write $B=A^{-1}$, and $A^{-1}=\left[R:_{S} A\right]=\{x \in S: x A \subseteq R\}[7$, Remarks 1.10, p. 90].

For a ring $S$, and an additive totally ordered abelian group $\Gamma$, let $\Gamma \cup \infty=$ $\Gamma \cup\{\infty\}$, where $\infty+g=g+\infty=\infty$ for all $g \in \Gamma \cup \infty$, and $g<\infty$ for all $g \in \Gamma$. A valuation on $S$ with values in $\Gamma$ is a map $v: S \longrightarrow \Gamma \cup \infty$ such that:

(1) $v(x y)=v(x)+v(y)$ for all $x, y \in S$.

(2) $v(x+y) \geqslant \min \{v(x), v(y)\}$ for all $x, y \in S$.

(3) $v(1)=0$ and $v(0)=\infty$.

The set $V=\{x \in S: v(x) \geqslant 0\}$ is called a valuation subring of $S$. If $v(S)=\{0, \infty\}$, then $v$ is said to be trivial, otherwise $v$ is called non-trivial. If $v(S)=\Gamma \cup \infty$, then $v$ is called a Manis valuation on $S$ and $V=\{x \in S: v(x) \geqslant 0\}$ is called a Manis subring of $S$.

For a subring $R$ of $S$, if there exists a Manis valuation $v: S \longrightarrow \Gamma \cup \infty$ such that $R=\{x \in S: v(x) \geqslant 0\}$, then the extension $R \subseteq S$ is called a Manis extension. In this case, $(R, \mathfrak{p})$ is called a Manis pair in $S$, where $\mathfrak{p}=\{x \in S: v(x)>0\}$. The ring $S$ is called a Prüfer extension of $R$ if $\left(R_{[\mathfrak{p}]}, \mathfrak{p}_{[\mathfrak{p}]}\right)$ is a Manis pair in $S$ for every maximal ideal $\mathfrak{p}$ of $R$. In this case, we say that $R$ is Prüfer in $S$. More on Manis valuations and Prüfer extensions can be found in [7]. The ring extension $R \subseteq S$ is called (weak) Prüfer star-multiplication extension $(P \star M E)$ if $\left(R_{[m]}, m_{[m]}\right)$ is a Manis pair in $S$ for every ( $S$-regular) $\star$-maximal ideal $m$ of $R$ [10, Defintion 3.1]. 
Remark 1.4. If $R \subseteq S$ is a Manis extension, then $R[X] \subseteq S[X]$ is Manis extension, where $X$ is an indeterminate over $S$. In fact, suppose that $v: S \rightarrow \Gamma \cup\{\infty\}$ is a valuation map such that $v(S)=\Gamma \cup\{\infty\}$ and $R=\{a \in S: v(a) \geqslant 0\}$. Denote by $\widetilde{v}$ the extension of $v$ to $S[X]$. The map $\widetilde{v}: S[X] \rightarrow \Gamma \cup\{\infty\}$ is defined by $\widetilde{v}\left(\sum_{i=1}^{\ell} a_{i} X^{i}\right)=\inf \left\{v\left(a_{i}\right): 1 \leqslant i \leqslant \ell\right\}$, where $a_{i} \in S$ for $1 \leqslant i \leqslant \ell$. Then by [8, Remarks(b), p.130], $\widetilde{v}$ is a valuation map. Furthermore, by the definition of $\widetilde{v}$, we have $\widetilde{v}(S[X])=\Gamma \cup\{\infty\}$ and $R[X]=\{f \in S[X]: \widetilde{v}(f) \geqslant 0\}$. It follows that the extension $R[X] \subseteq S[X]$ is Manis.

In this paper, we prove several results on localization (in the ring extension context), and we discuss associated polynomial ring extensions, Nagata rings, star invertibility, and Prüfer star-multiplication extensions. Many results in this article have well-known origins in the domain case (see for example [6]). In Section 2, we discuss some properties of ring extensions. These properties will be needed in the proofs of results presented in Section 3.

In Section 3, we establish some results on star operations. These properties are observations made while working on other projects (see for example [9], [10], [12]). Their proofs are contributions to a better understanding of the structures of star operations on ring extensions. In Theorem 3.13, we show that for each maximal ideal $\mathfrak{m}$ of $R$, the extension $R_{[\mathfrak{m}]} \subseteq S$ is Manis if and only $R[X]_{[\mathfrak{m} R[X]]} \subseteq$ $S[X]$ is a Manis extension whenever $R[X] \subseteq S[X]$ is integrally closed and $X$ is an indeterminate over $S$. In Theorem 3.15, we study Prüfer star-multiplicative extension in pullback diagrams of type $\square$.

\section{Preliminaries}

For a ring extension $R \subseteq S$ and an $R$-submodule $M$ of $S$, if $\tau$ is a multiplicatively closed subset of $R$, we denote by $M_{[\tau]}$ the set of all $x \in S$ such that $t x \in M$ for some $t \in \tau$. The set $M_{[\tau]}$ is an $R$-submodule of $S$. In particular, $R_{[\tau]}$ is a ring satisfying $R \subseteq R_{[\tau]} \subseteq S$ (see comment after [7, Definition 10, p. 18]). If $\mathfrak{p}$ is a prime ideal of $R$, and $\tau=R \backslash \mathfrak{p}$, then $M_{[\mathfrak{p}]}$ denotes the set of all $x \in S$ such that $t x \in M$ for some $t \in \tau$.

Remark 2.1. Let $R \subseteq S$ be a ring extension, and let $N$ be a multiplicatively closed subset of $R$. Let $\mathfrak{p}$ be a prime ideal of $R$ such that $\mathfrak{p} \cap N=\varnothing$. Then

(1) The set $\mathfrak{p}_{[N]}$ is a prime ideal of $R_{[N]}$ and $\mathfrak{p}_{[N]} \cap N=\varnothing$.

(2) If $I$ is an ideal of $R$ such that $I_{[N]} \subseteq \mathfrak{p}_{[N]}$, then $I \subseteq \mathfrak{p}$. 
Proof. (1) Let $x, y \in R_{[N]}$ such that $x y \in \mathfrak{p}_{[N]}$. Then there exist $t, t_{1}, t_{2} \in N$ such that $t_{1} x \in R, t_{2} y \in R$ and $t x y \in \mathfrak{p}$. It follows that $t\left(t_{1} x\right)\left(t_{2} y\right)=\left(t_{1} t_{2}\right)(t x y) \in \mathfrak{p}$. But $t \notin \mathfrak{p}$ since $\mathfrak{p} \cap N=\varnothing$. Therefore $\left(t_{1} x\right)\left(t_{2} y\right)=\left(t_{1} t_{2}\right)(t x y) \in \mathfrak{p}$. Hence $t_{1} x \in \mathfrak{p}$ or $t_{2} y \in \mathfrak{p}$. Thus $x \in \mathfrak{p}_{[N]}$ or $y \in \mathfrak{p}_{[N]}$. This shows that $\mathfrak{p}_{[N]}$ is prime ideal of $R_{[N]}$.

We show that $\mathfrak{p}_{[N]} \cap N=\varnothing$. By contradiction, suppose that $\mathfrak{p}_{[N]} \cap N \neq \varnothing$. Let $t_{1} \in P_{[N]} \cap N$. Then there exists $t_{2} \in N$ such that $t_{1} t_{2} \in \mathfrak{p}$. Furthermore, $t_{1} t_{2} \in N$ since $N$ is multiplicatively closed. It follows that $t_{1} t_{2} \in P \cap N$. Which is a contradiction since $\mathfrak{p} \cap N=\varnothing$. This shows that $\mathfrak{p}_{[N]} \cap N=\varnothing$.

(2) Let $a \in I$. Then $a \in I \subseteq I_{[N]} \subseteq \mathfrak{p}_{[N]}$. Hence, there exists $t \in N$ such that $t a \in \mathfrak{p}$. But $t \notin \mathfrak{p}$ since $\mathfrak{p} \cap N=\varnothing$. It follows that $a \in \mathfrak{p}$ since $\mathfrak{p}$ is prime. This shows that $I \subseteq \mathfrak{p}$.

Remark 2.2. Let $R \subseteq S$ be a ring extension, and let $N$ be a multiplicatively closed subset of $R$. Let $J$ be a proper ideal of the ring $R_{[N]}$. Then

(1) $(J \cap R)_{[N]}=J_{[N]}$.

(2) If $J$ is prime ideal of $R_{[N]}$ and $J \cap N=\varnothing$, then $J=J_{[N]}$.

(3) If $J$ is prime ideal of $R_{[N]}$ and $J \cap N \neq \varnothing$, then $J_{[N]}=R_{[N]}$

Proof. (1) We show that $(J \cap R)_{[N]}=J_{[N]}$. Let $w \in(J \cap R)_{[N]}$. Then $t w \in$ $J \cap R \subseteq J$ for some $t \in N$. Hence $w \in J_{[N]}$. This shows that $(J \cap R)_{[N]} \subseteq J_{[N]}$. On the other hand, if $u$ is an element of $J_{[N]}$, then there exists $t \in N$ such that $t u \in J \subseteq R_{[N]}$. So there exists $t^{\prime} \in N$ such that $t^{\prime}(t u) \in R$. It follows that $\left(t^{\prime} t\right) u \in J \cap R$. Thus $u \in(J \cap R)_{[N]}$ since $t t^{\prime} \in N$. Therefore, $J_{[N]} \subseteq(J \cap R)_{[N]}$. This shows that $(J \cap R)_{[N]}=J_{[N]}$.

(2) Suppose that $J$ is a prime ideal of $R_{[N]}$ such that $J \cap N=\varnothing$. We show that $J_{[N]} \subseteq J$ since the containment $J \subseteq J_{[N]}$ is always true. Let $x \in J_{[N]}$. Then $t x \in J$ for some $t \in N$. But $t \notin J$ since $J \cap N=\varnothing$. It follows that $x \in J$ since $J$ is prime. This shows that $J=J_{[N]}$.

(3) Suppose that $J$ is a prime ideal of $R_{[N]}$ such that $J \cap N \neq \varnothing$. Let $t \in J \cap N$. Then $t(1) \in J \cap N$. Hence, $1 \in(J \cap N)_{[N]}$. Therefore, $(J \cap N)_{[N]}=R_{[N]}$. It follows from (1) that $J_{[N]}=R_{[N]}$.

Lemma 2.3. Let $R \subseteq S$ be a ring extension, and let $N$ be a multiplicatively closed subset of $R$. Let $\mathcal{P}=\{\mathfrak{p}: \mathfrak{p}$ is a prime ideal of $R$ and $p \cap N=\varnothing\}$ and $\mathcal{M}=$ $\{\mathfrak{m}: \mathfrak{m}$ is a maximal ideal of $R$ and $\mathfrak{m} \cap N=\varnothing\}$.

(1) For each $\mathfrak{p} \in \mathcal{P}, \mathfrak{p}_{[N]}$ is a prime ideal of $R_{[N]}$ satisfying $\mathfrak{p}_{[N]} \cap N=\varnothing$.

(2) For each $\mathfrak{m} \in \mathcal{M}, \mathfrak{m}_{[N]}$ is a maximal ideal of $R_{[N]}$ satisfying $\mathfrak{m}_{[N]} \cap N=\varnothing$. 
Proof. (1) By Remark 2.1(1), each element of the set $\left\{\mathfrak{p}_{[N]}: \mathfrak{p} \in \mathbb{P}\right\}$ is a prime ideal of $R_{[N]}$ which does not intercept with $N$. Let $J$ be a proper prime ideal of $R_{[N]}$ such that $J \cap N=\varnothing$, and let $\mathfrak{p}_{0}=J \cap R$. Then by Remark 2.2(2), $J=\mathfrak{p}_{0[N]}$. Hence $J \in\left\{\mathfrak{p}_{[N]}: \mathfrak{p} \in \mathbb{P}\right\}$.

(2) Let $\mathfrak{m}$ be an element of $\mathcal{M}$. By Remark 2.1(1), $\mathfrak{m}_{[N]}$ is a prime ideal of $R_{[N]}$ and $\mathfrak{m}_{[N]} \cap N=\varnothing$. Suppose that $J_{0}$ is a maximal ideal of $R_{[N]}$ such that $\mathfrak{m}_{[N]} \subseteq J_{0}$. Then $\mathfrak{m} \subseteq J_{0} \cap R$. It follows from the maximality of $\mathfrak{m}$ that $\mathfrak{m}=J_{0} \cap R$ or $J_{0} \cap R=R$. If $J_{0} \cap R=R$, then $1 \in J_{0}$. Which is a contradiction since $J_{0} \subsetneq R_{[N]}$. Thus $\mathfrak{m}=J_{0} \cap R$. Hence $\mathfrak{m}_{[N]}=\left(J_{0} \cap R\right)_{[N]}$. Suppose that $J_{0} \cap N \neq \varnothing$. Let $t \in J_{0} \cap N \subseteq J_{0} \cap R$. Then $t(1) \in J_{0} \cap R$. Hence $1 \in\left(J_{0} \cap R\right)_{[N]}=\mathfrak{m}_{[N]}$. Which is a contradiction since $\mathfrak{m}_{[N]}$ is a proper ideal of $R_{[N]}$. This shows that $J_{0} \cap N=\varnothing$. It follows from Remark 2.2(2) that $\mathfrak{m}_{[N]}=\left(J_{0} \cap R\right)_{[N]}=J_{0[N]}=J_{0}$. This shows that $\mathfrak{m}_{[N]}$ is a maximal ideal of $R_{[N]}$.

Let $R \subseteq S$ and $L \subseteq T$ be two ring extensions, and consider the following commutative diagram

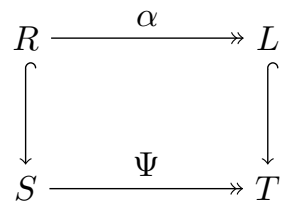

where $\operatorname{ker} \Psi$ is an ideal of $R, \Psi: S \longrightarrow T$ is surjective, the restriction $\alpha: R \longrightarrow L$ of $\Psi$ is also surjective and the vertical mappings are inclusions. When $\operatorname{ker} \Psi$ is a maximal ideal of $S$, the previous commutative diagram is called a pullback diagram of type $\square$. Pullback diagrams of type $\square$ are studied by S. Gabelli and E. Houston in [2].

Lemma 2.4. Consider a pullback diagram of type $\square$. Let $A$ be an $R$-submodule of $S$, and let $B$ be an L-submodule of $T$.

(1) If $A S=S$, then $\operatorname{ker} \Psi \subseteq A$.

(2) Suppose that $\operatorname{ker} \Psi \subseteq \operatorname{Jac}(R)$. If $A=\Psi(B)$ and $B T=T$, then $A S=S$.

(3) If $A S=B S$ and $\Psi(A)=\Psi(B)$, then $A=B$.

Proof. The statement (1) is given by [11, Remark 1.1], and the statement (2) is given by [11, Lemma 2.8].

For the proof of the statement (3), suppose that $A S=B S$. Then by (1), ker $\Psi \subseteq A$ and ker $\Psi \subseteq B$. It follows from [9, Remark 2.5] that $A \subseteq B$ and $B \subseteq A$. Thus $A=B$. 
Lemma 2.5. Consider a pullback diagram of type $\square$. Then $R$ is integrally closed in $S$ if and only if $L$ is integrally closed in $T$.

Proof. Suppose that $R$ is integrally closed in $S$. Let $v \in T$, and let $g(X)=$ $\sum_{i=0}^{\ell} b_{i} X^{i} \in L[X]$ with $b_{i} \in L$ for $1 \leqslant i \leqslant \ell$ such that $g(v)=0$, where $X$ is an indeterminate over $S$. Since $\Psi$ is surjective and $L=\Psi(R)$, there exist $u \in S$ and $a_{0}, \ldots, a_{\ell} \in R$ such that $v=\Psi(u), b_{0}=\Psi\left(a_{0}\right), \ldots, b_{\ell}=\Psi\left(a_{\ell}\right)$. Let $f(X)=$ $\sum_{i=0}^{\ell} a_{i} X^{i}$. Then $f(X) \in R[X]$ and $\Psi(f(u))=\sum_{i=0}^{\ell} \Psi\left(a_{i}\right) \Psi(u)^{i}=\sum_{i=0}^{\ell} b_{i} v^{i}=g(v)=0$. This shows that $f(u) \in \operatorname{ker} \Psi \subseteq R$. So there exists $r \in R$ such that $f(u)=r$. Let $h(X)=f(X)-r$. Then $h(X) \in R[X]$, and $h(u)=0$. Therefore $u \in R$ since $R$ is integrally closed in $S$. It follows that $v=\Psi(u) \in L$. This shows that $L$ is integrally closed in $T$.

Conversely, suppose that $L$ is integrally closed in $T$. Let $u \in S$, and let $f(X)=$ $\sum_{i=0}^{\ell} a_{i} X^{i} \in R[X]$ be a polynomial with $a_{i} \in R$ for $1 \leqslant i \leqslant \ell$ such that $f(u)=0$. Let $g(X)=\sum_{i=0}^{\ell} \Psi\left(a_{i}\right) X^{i} \in L[X]$. Then $g(\Psi(u))=\sum_{i=0}^{\ell} \Psi\left(a_{i}\right) \Psi(u)^{i}=\sum_{i=0}^{\ell} \Psi\left(a_{i} u^{i}\right)=$ $\Psi\left(\sum_{i=0}^{\ell} a_{i} u^{i}\right)=\Psi(f(u))=0$. Thus $\Psi(u) \in L$, since $L$ is integrally closed in $T$. Since $L=\Psi(R)$, there exists $v \in R$ such that $\Psi(u)=\Psi(v)$. Hence $u-v \in \operatorname{ker} \Psi \subseteq R$. It follows that $u \in R$ since $v \in R$. This proves that $R$ is integrally closed in $S$.

For the rest of the article, if $R \subseteq S$ is a ring extension, and $X$ is an indeterminate over $S$, then for any multiplicatively closed subset $N$ of $R[X], R[X]_{[N]}$ denotes the set of elements $f \in S[X]$ such that $h f \in R[X]$ for some $h \in N$.

Lemma 2.6. Let $R \subseteq S$ be a ring extension, and let $T$ be a multiplicatively closed subset of $R$.

(1) $R[X]_{[T[X]]}=R_{[T]}[X]$, where $X$ is an indeterminate over $S$.

(2) If $N$ is a multiplicatively closed subset of $R$ such that $N \subseteq T$, and $A$ is an $R$-submodule of $S$, then $T_{[N]}$ is a multiplicatively closed subset of $R_{[N]}$ and $\left(A_{[N]}\right)_{\left[T_{[N]}\right]}=A_{[T]}$.

Proof. (1) Let $f=\sum_{i=1}^{k} a_{i} X^{i} \in R[X]_{[T[X]]}$ with $a_{i} \in S$ for $1 \leqslant i \leqslant k$. Then $h f \in R[X]$ for some $h \in T[X]$. Thus $c_{R}(h f) \subseteq R$. Hence $c_{R}(h f) c_{R}(h)^{\ell} \subseteq R$ for any positive integer $\ell$. It follows from the Dedeking-Merteens formula ([8, Theorem 1.1, p. 126]) that $c_{R}(f) c_{R}(h)^{n} \subseteq R$ for some positive integer $n$. But $c_{R}(h)^{n} \subseteq T$ since $T$ is multiplicatively closed. Let $u \in c_{R}(h)^{n}$. Then $u a_{i} \in R$ for $1 \leqslant i \leqslant k$. Hence $a_{i} \in R_{[T]}$. It follows that $f \in R_{[T]}[X]$. This shows that $R[X]_{T[X]} \subseteq R_{[T]}[X]$. 
Let $f=\sum_{i=1}^{k} a_{i} X^{i} \in R_{[T]}[X]$ with $a_{i} \in R_{[T]}$ for $1 \leqslant i \leqslant k$. Then for $1 \leqslant i \leqslant k$, there exists $t_{i} \in T$ such that $t_{i} a_{i} \in R$. Let $t=\prod_{i=1}^{k} t_{i}$. Then $t \in T \subseteq T[X]$ and $t f \in R[X]$. This shows that $f \in R[X]_{[T[X]]}$. Hence $R_{[T]}[X] \subseteq R[X]_{[T[X]]}$. Therefore $R_{[T]}[X]=R[X]_{[T[X]]}$.

(2) Let $u_{1}, u_{2} \in T_{[N]}$. Then $s_{1} u_{1} \in T$ and $s_{2} u_{2} \in T$ for some $s_{1}, s_{2} \in N$. Thus $s_{1} s_{2}\left(u_{1} u_{2}\right)=\left(s_{1} u_{1}\right)\left(s_{2} u_{2}\right) \in T$. It follows that $u_{1} u_{2} \in T_{[N]}$. This shows that $T_{[N]}$ is multiplicatively closed. Let $x \in\left(A_{[N]}\right)_{\left[T_{[N]}\right]}$. Then $u x \in A_{[N]}$ for some $u \in T_{[N]}$. Let $s, s^{\prime} \in N$ such that $s u \in T$ and $s^{\prime}(u x) \in A$. Then $s^{\prime}(s u) x=\left(s s^{\prime}\right)(u x) \in A$. It follows that $x \in A_{[T]}$ since $s^{\prime}(s u) \in T$. This shows that $\left(A_{[N]}\right)_{\left[T_{[N]}\right]} \subseteq A_{[T]}$.

Let $x \in A_{[T]}$. Then $t x \in A \subseteq A_{[N]}$ for some $t \in T$. But $T \subseteq T_{[N]}$. Hence $x \in\left(A_{[N]}\right)_{\left[T_{[N]}\right]}$, and so $A_{[T]} \subseteq\left(A_{[N]}\right)_{\left[T_{[N]}\right]}$. Therefore, $A_{[T]}=\left(A_{[N]}\right)_{\left[T_{[N]}\right]}$.

\section{Some properties of star operations on ring extensions}

In this section, we establish some results on star operations. Let $\star: \mathcal{J}(R, S) \longrightarrow$ $\mathcal{J}(R, S)$ be a star operation on a ring extension $R \subseteq S$. An $R$-submodule $A$ of $R$ is said to be $\star$-invertible if $\left(A A^{-1}\right)^{\star}=R^{\star}$. In the next result, we give a necessary condition for a finitely generated $S$-regular $R$-submodule of $S$ to be $\star$-invertible.

Proposition 3.1. Let $R \subseteq S$ be a ring extension, and let $\star: \mathcal{J}(R, S) \longrightarrow \mathcal{J}(R, S)$ be a star operation, and let $A$ be an $S$-regular $R$-submodule of $S$. If $A$ is $\star$-invertible, then for each $\star$-maximal ideal $\mathfrak{m}$ of $R$, we have $A_{[\mathfrak{m}]}=(a)_{[\mathfrak{m}]}$ for some $a \in A$.

Proof. Let $A$ be an $S$-regular $R$-submodule of $S$. Suppose that $A$ is $\star$-invertible, and let $\mathfrak{m}$ be a $\star$-maximal ideal of $R$. Then $\left(A A^{-1}\right)^{\star}=R^{\star}$. Hence $A A^{-1} \nsubseteq$ $\mathfrak{m}$, otherwise $R \subseteq R^{\star} \subseteq\left(A A^{-1}\right)^{\star} \subseteq \mathfrak{m}$. This is a a contradiction. Therefore, there exists $t \in A A^{-1} \subseteq R$ such that $t \notin \mathfrak{m}$. Let $x \in R_{[\mathfrak{m}]}$. Then there exists $s \in R \backslash \mathfrak{m}$ such that $s x \in R$. Thus $(t s) x=t(s x) \in A A^{-1}$. It follows that $x \in$ $\left(A A^{-1}\right)_{[\mathfrak{m}]}$ since $t s \notin \mathfrak{m}$. This shows that $R_{[\mathfrak{m}]} \subseteq\left(A A^{-1}\right)_{[\mathfrak{m}]}$, and so $\left(A A^{-1}\right)_{[\mathfrak{m}]}=$ $R_{[\mathfrak{m}]}$. Hence $\left(\left(A A^{-1}\right)_{[\mathfrak{m}]}\right)_{\mathfrak{m}_{[\mathfrak{m}]}}=\left(R_{[\mathfrak{m}]}\right)_{\mathfrak{m}_{[\mathfrak{m}]}}$. But by [7, lemma 2.9(b), p.28], we have $\left(\left(A A^{-1}\right)_{[\mathfrak{m}]}\right)_{\mathfrak{m}_{[\mathfrak{m}]}}=\left(A A^{-1}\right)_{\mathfrak{m}}$ and $\left(R_{[\mathfrak{m}]}\right)_{\mathfrak{m}_{[\mathfrak{m}]}}=R_{\mathfrak{m}}$. Hence, $A_{\mathfrak{m}} A_{\mathfrak{m}}^{-1}=$ $\left(A A^{-1}\right)_{\mathfrak{m}}=R_{\mathfrak{m}}$. Thus $A_{\mathfrak{m}}$ is a locally principal $R_{\mathfrak{m}}$-submodule of $S_{\mathfrak{m}}$ [7, Proposition 2.3, p. 97]. In particular, $A_{\mathfrak{m}}$ is a principal $R_{\mathfrak{m}}$-submodule of $S_{\mathfrak{m}}$. So there exists $a \in R$ and $s \in R \backslash \mathfrak{m}$ such that $A_{\mathfrak{m}}=\left(\frac{a}{s}\right) R_{\mathfrak{m}}=(a) R_{\mathfrak{m}}=(a)_{\mathfrak{m}}$. Now we show that $A_{[\mathfrak{m}]}=(a)_{[\mathfrak{m}]}=(a) R_{[\mathfrak{m}]}$. Let $y \in A_{[\mathfrak{m}]}$, and let $j: S \rightarrow S_{\mathfrak{m}}$ be the map defined by $j(x)=\frac{x}{1}$. Then by $\left[7\right.$, definition 10, p. 18], $A_{[\mathfrak{m}]}=j^{-1}\left(A_{\mathfrak{m}}\right)$. It follows that $j(y) \in A_{\mathfrak{m}}$. Thus $\frac{y}{1} \in(a)_{\mathfrak{m}}$. Therefore, $\frac{y}{1}=\frac{r a}{t}$ for some $r \in R$, and $t \in A \backslash \mathfrak{m}$. So, 
$(s t) y=(s r) a \in(a)$ for some $s \in A \backslash \mathfrak{m}$. It follows that $y \in(a)_{[\mathfrak{m}]}$ since $(s t) \in A \backslash \mathfrak{m}$. This shows that $A_{[\mathfrak{m}]} \subseteq(a)_{[m]}$. On the other hand, let $z \in(a)_{[\mathfrak{m}]}$. Then there exists $t_{1} \in R \backslash \mathfrak{m}$ such that $t_{1} z=r a \in A_{\mathfrak{m}}$ for some $r \in R$. Hence there exists $c \in A$ and $t_{2} \in R \backslash \mathfrak{m}$ such that $t_{1} z=\frac{c}{t_{1}}$. Thus $\left(t_{1} t_{2} t_{3}\right) z=c t_{3} \in A$ for some $t_{3} \in R \backslash \mathfrak{m}$. Thus $z \in A_{[\mathfrak{m}]}$ since $t_{1} t_{2} t_{3} \in R \backslash \mathfrak{m}$. This shows that $(a)_{[\mathfrak{m}]} \subseteq A_{[\mathfrak{m}]}$. Therefore, $A_{[\mathfrak{m}]}=(a)_{[\mathfrak{m}]}$.

Lemma 3.2. Let $\star$ be a strict star operation of finite type on a ring extension $R \subseteq S$, and let $A$ be an $R$-submodule of $S$ such that $A^{\star}=A$. Then for any multiplicatively closed subset $\tau$ of $R$, we have $\left(A_{[\tau]}\right)^{\star}=A_{[\tau]}$. In particular, $\left(R_{[\tau]}\right)^{\star}=R_{[\tau]}$.

Proof. Let $A$ be an $R$-submodule of $S$ such that $A^{\star}=A$. Let $x \in\left(A_{[\tau]}\right)^{\star}$. Since $\star$ is of finite type, there exists a finitely generated $R$-module $J$ contained in $A_{[\tau]}$ such that $x \in J^{\star}$. Let $u_{1}, \ldots, u_{\ell} \in A_{[\tau]}$ such that $J=\left(u_{1}, \ldots, u_{\ell}\right) R$. For $1 \leqslant i \leqslant \ell$, there exists $t_{i} \in \tau$ such that $t_{i} u_{i} \in A$. Let $t=\prod_{i=1}^{\ell} t_{i}$. Then $t J \subseteq A$. Hence $t x \in t J^{\star} \subseteq(t J)^{\star} \subseteq A^{\star}=A$. Therefore $x \in A_{[\tau]}$. This shows that $\left(A_{[\tau]}\right)^{\star} \subseteq A_{[\tau]}$. Then $\left(A_{[\tau]}\right)^{\star}=A_{[\tau]}$ since the containment $A_{[\tau]} \subseteq\left(A_{[\tau]}\right)^{\star}$ is always true.

In the next remark, we give an example of a star operation $\star_{1}$ on a ring extension $R \subseteq S$, and a star operation $\star_{2}$ on the extension $R[X] \subseteq S[X]$ satisfying the condition $A^{\star_{1}} R[X]=(A R[X])^{\star_{2}}$ for each $R$-submodule $A$ of $S$, where $X$ is an indeterminate over $S$. This condition will be assumed in many of the results established in this section.

Remark 3.3. ([12, Remark 3.7]) Let $R \subseteq S$ be a ring extension, and let $X$ be an indeterminate over $S$. For each $R$-submodule $A$ of $S$, write $A^{\star_{1}}=\left(R:_{S}\left(R:_{S} A\right)\right)$, and for each $R[X]$-submodule $M$ of $S[X]$, write

$$
M^{\star_{2}}=\left(R[X]:_{S[X]}\left(R[X]:_{S[X]} M\right)\right) .
$$

Then $\star_{1}$ is a strict star on the extension $R \subseteq S$, and $\star_{2}$ is a strict star operation on the extension $R[X] \subseteq S[X]$ satisfying $A^{\star_{1}} R[X]=(A R[X])^{\star_{2}}$ for each $R$-submodule $A$ of $S$.

Proposition 3.4. Let $\star_{1}$ be a star operation of finite type on a ring extension, and let $\star_{2}$ be a star extension of finite type on $R[X] \subseteq S[X]$, where $X$ is an indeterminate over $S$. Let $T$ be a multiplicatively closed subset of $R[X]$. If $I$ is an ideal of $R$ such that $I^{\star_{1}} R[X]=(I R[X])^{\star_{2}}$, then $\left(I^{\star_{1}}[X]\right)_{[T]}=\left(I[X]_{[T]}\right)^{\star_{2}}$. 
Proof. Let $f \in\left(I[X]_{[T]}\right)^{\star_{2}}$. Since $\star_{2}$ is of finite type, there exist $f_{1}, \ldots, f_{n} \in$ $I[X]_{[T]}$ such that $f \in\left(f_{1}, \ldots, f_{n}\right)^{\star_{2}}$. Let $t_{i} \in T$ such that $t_{i} f_{i} \in I[X]$ for $1 \leqslant i \leqslant n$, and let $t=\prod_{i=1}^{n} t_{i}$. Then $t f_{i} \in I[X]$. Hence $t f \in t\left(f_{1}, \ldots, f_{n}\right)^{\star_{2}} \subseteq\left(t f_{1}, \ldots, t f_{n}\right)^{\star_{2}} \subseteq$ $(I[X])^{\star_{2}}=I^{\star_{1}}[X]$. It follows that $f \in\left(I^{\star_{1}}[X]\right)_{[T]}$. This shows that $\left(I[X]_{[T]}\right)^{\star_{2}} \subseteq$ $\left(I^{\star_{1}}[X]\right)_{[T]}$.

For the other containment, let $f \in\left(I^{\star_{1}}[X]\right)_{[T]}$. Then $t f \in I^{\star_{1}}[X] \subseteq(I[X])^{\star_{2}} \subseteq$ $\left(I[X]_{[T]}\right)^{\star_{2}}$ for some $t \in T$. Thus $f \in\left(\left(I[X]_{[T]}\right)^{\star_{2}}\right)_{[T]}$. But by Lemma 3.2, $\left(I[X]_{[T]}\right)^{\star_{2}} \subseteq\left(R[X]_{[T]}\right)^{\star_{2}}=R[X]_{[T]}$. Furthermore, $R[X]_{[T]}$ is an overring of $R[X]$ in $S[X]$ (see comment after [7, Definition, p. 18]). It follows from [8, Proposition 4.2 , p. 146] that $\left(I[X]_{[T]}\right)^{\star_{2}} \in \mathcal{J}\left(\left(R_{[T]}[X]\right)^{\star_{2}}, S[X]\right)$. But by Lemma 3.2 we have $\left(R_{[T]}[X]\right)^{\star_{2}}=R_{[T]}[X]$. Therefore, $\left(I[X]_{[T]}\right)^{\star_{2}} \in \mathcal{J}\left(\left(R_{[T]}[X]\right), S[X]\right)$. Hence $\left(I[X]_{[T]}\right)^{\star_{2}}$ is an ideal of $R[X]_{[T]}$. Therefore, from Remark 2.2 we have $\left(\left(I[X]_{[T]}\right)^{\star_{2}}\right)_{[T]}=\left(I[X]_{[T]}\right)^{\star_{2}}$. Hence $f \in\left(I[X]_{[T]}\right)^{\star_{2}}$, and so $\left(I^{\star_{1}}[X]\right)_{[T]} \subseteq$ $\left(I[X]_{[T]}\right)^{\star_{2}}$. Thus $\left(I^{\star_{1}}[X]\right)_{[T]}=\left(I[X]_{[T]}\right)^{\star_{2}}$.

For the rest of this article, if $\star$ is a star operation on a ring extension $R \subseteq S$ and $X$ is an indeterminate over $S$, then we denote by $\operatorname{Max}(\star)$ the set of all $\star$-maximal ideals of $R$, and $N(\star)=\left\{f \in R[X]: c_{R}(f)^{\star}=R^{\star}\right\}$.

Lemma 3.5. Let $\star$ be a star operation on a ring extension $R \subseteq S$, and let $X$ be an indeterminate over $S$. The set $N(\star)$ is multiplicatively closed subset of $R[X]$.

Proof. Let $f, g \in N(\star)$. Then $c_{R}(f)^{\star}=R^{\star}$ and $c_{R}(g)^{\star}=R^{\star}$. By the DedekindMertens formula [8, Theorem 1.1, p. 126], we have $c_{R}(f g) c_{R}(g)^{n}=c_{R}(f) c_{R}(g)^{n+1}$ for some positive integer $n$. Thus $\left(c_{R}(f g) c_{R}(g)^{n}\right)^{\star}=\left(c_{R}(f) c_{R}(g)^{n+1}\right)^{\star}$. Furthermore, for any positive integer $\ell$, we have $\left(R^{\star}\right)^{\ell}=R^{\star}$ since $R^{\star}$ is a ring [8, Propisition 4.2 (b), p. 146]. But by [8, Proposition 4.1 (a), p. 146] and Remark 1.2 that $\left(c_{R}(f g) c_{R}(g)^{n}\right)^{\star}=\left(c_{R}(f g)^{\star}\left(c_{R}(g)^{n}\right)^{\star}\right)^{\star}=\left(c_{R}(f g)^{\star} R^{\star}\right)^{\star}=c_{R}(f g)^{\star}$ and $\left(c_{R}(f) c_{R}(g)^{n+1}\right)^{\star}=\left(c_{R}(f)^{\star}\left(c_{R}(g)^{n+1}\right)^{\star}\right)^{\star}=\left(R^{\star} R^{\star}\right)^{\star}=R^{\star}$. Hence $c_{R}(f g)^{\star}=R^{\star}$. This shows that $f g \in N(\star)$.

For the rest of the article, we denote by $d: \mathcal{J}(R, S) \longrightarrow \mathcal{J}(R, S)$ the identity and $N(d)=\left\{f \in R[X]: c_{R}(f)=R\right\}$, where $X$ is an indeterminate over the ring extension $R \subseteq S$.

Proposition 3.6. Let $R \subseteq S$ be a ring extension, and let $X$ be an indeterminate over $S$. Then the set $N(d)$ contains no zero divisors of $R[X]$.

Proof. By contradiction, suppose that there exists $f \in N(d)$ which is a zero divisor of $R[X]$. Then $c_{R}(f)=R$ and there exists a nonzero $g \in R[X]$ such that $f g=0$. 
Hence $c_{R}(f g)=0$. Furthermore, by the Dedekind-Mertens formula ([8, Theorem 1.1, p. 126]), we have $c_{R}(g) c_{R}(f)^{n+1}=c_{R}(f g) c_{R}(f)^{n}=0$. It follows that $c_{R}(g) R=$ $c_{R}(g)=0$. This is a contradiction since $g \neq 0$. This shows that $N(d)$ has no zero divisors.

The following remark is an analog of [6, Proposition 2.1]; which itself originates from [3, Proposition 33.1, p. 410].

Proposition 3.7. Let $\star$ be a strict star operation of finite type on a ring extension $R \subseteq S$, and let $X$ be an indeterminate over $S$. Then

(1) $N(\star)=R[X] \backslash \bigcup_{\mathfrak{m} \in \operatorname{Max}(\star)}(\mathfrak{m}[X])$.

(2) For each $\star$-maximal ideal $\mathfrak{m}$ of $R, \mathfrak{m}[X]_{[N(\star)]}$ is a maximal ideal of $R[X]_{[N(\star)]}$. Furthermore, $\mathfrak{m}_{[N(\star)]} \cap N(\star)=\varnothing$.

Proof. (1) Let $f \in N(\star)$. Then $c_{R}(f)^{\star}=R$. Thus for each $\mathfrak{m} \in \operatorname{Max}(\star)$, we have $c_{R}(f) \nsubseteq \mathfrak{m}$. Otherwise, we will get $R \subseteq R^{\star}=c_{R}(f) \subseteq \mathfrak{m}^{\star}=\mathfrak{m}$; which is a contradiction. This shows that $f \notin \mathfrak{m}[X]$ for each $\mathfrak{m} \in \operatorname{Max}(\star)$. Hence $f \in$ $R[X] \backslash \bigcup_{\mathfrak{m} \in \operatorname{Max}(\star)} \mathfrak{m}[X]$. This shows that $N(\star) \subseteq R[X] \backslash \bigcup_{\mathfrak{m} \in \operatorname{Max}(\star)}(\mathfrak{m}[X])$.

On the other hand, let $f \in R[X] \backslash \bigcup_{\mathfrak{m} \in \operatorname{Max}(\star)}(\mathfrak{m}[X])$. By contradiction, suppose that $c_{R}(f)^{\star} \subsetneq R$. Then $c_{R}(f)^{\star}$ is a proper ideal of $R$. Furthermore, by [8, Proposition 4.6, p. 149], $\left(c_{R}(f)^{\star} \cap R\right)^{\star}=c_{R}(f)^{\star} \cap R^{\star}=c_{R}(f)^{\star} \cap R$. So $c_{R}(f)^{\star} \cap R$ is a proper $\star$-ideal of $R$. It follows from Lemma 1.3 that $c_{R}(f)^{\star} \cap R$ is contained in a $\star$-maximal ideal $\mathfrak{m}_{0}$. Therefore, $c_{R}(f) \subseteq c_{R}(f)^{\star} \cap R \subseteq \mathfrak{m}_{0}$ of $R$. It follows that $f \in \mathfrak{m}_{0}[X]$. This is a contradiction since $f \in R[X] \backslash \bigcup_{\mathfrak{m} \in \operatorname{Max}(\star)}(\mathfrak{m}[X])$. Thus $c_{R}(f)^{\star}=R$.

(2) The proof follows from part (1) and Lemma 2.3(2).

Corollary 3.8. Let $R \subseteq S$ be a ring extension, and let $\star_{1}$ be a strict star operation of finite type on a ring extension $R \subseteq S$, and let $\star_{2}$ be a star operation on $R[X] \subseteq$ $S[X]$ such that $A^{\star_{1}} R[X]=(A R[X])^{\star_{2}}$ for each $R$-submodule $A$ of $S$, where $X$ is an indeterminate over $S$. Then for each $\star_{1}$-maximal ideal $\mathfrak{m}$ of $R, \mathfrak{m}[X]_{\left[N\left(\star_{1}\right)\right]}$ is $a \star_{2}$-maximal ideal of $R[X]_{\left[N\left(\star_{1}\right)\right]}$.

Proof. By Proposition $3.7(2), \mathfrak{m}[X]_{\left[N\left(\star_{1}\right)\right]}$ is a maximal ideal of $R[X]_{\left[N\left(\star_{1}\right)\right]}$. Furthermore, by Proposition 3.4, we have

$$
\left(\mathfrak{m}[X]_{\left[N\left(\star_{1}\right)\right]}\right)^{\star_{2}}=(\mathfrak{m}[X])_{\left[N\left(\star_{1}\right)\right]}^{\star_{2}}=\mathfrak{m}^{\star_{1}}[X]_{\left[N\left(\star_{1}\right)\right]}=\mathfrak{m}[X]_{\left[N\left(\star_{1}\right)\right]}
$$

This shows that $\mathfrak{m}[X]_{\left[N\left(\star_{1}\right)\right]}$ is a $\star_{2}$-maximal ideal of $R[X]_{\left[N\left(\star_{1}\right)\right]}$.

Proposition 3.9. Let $\star_{1}$ be a star operation on a ring extension $R \subseteq S$, and let $\star_{2}$ be a star operation on $R[X] \subseteq S[X]$, where $X$ is an indeterminate over $S$. Let $A$ 
be an $R$-submodule of $S$ such that $A^{\star_{1}} R[X]=(A R[X])^{\star_{2}}$. Then $A$ is $\star_{1}$-invertible if and only if $A R[X]$ is $\star_{2}$-invertible.

Proof. Let $A$ be an $R$-submodule of $S$ such that $A^{\star_{1}} R[X]=(A R[X])^{\star_{2}}$. Suppose that $A$ is $\star_{1}$-invertible. Then $\left(A\left[R:_{S} A\right]\right)^{\star_{1}}=R$. Thus $\left(A\left[R:_{S} A\right]\right)^{\star_{1}} R[X]=R[X]$. It follows from the hypothesis that

$$
\left(A R[X]\left[R:_{S} A\right] R[X]\right)^{\star_{2}}=\left(A\left[R:_{S} A\right] R[X]\right)^{\star_{2}}=\left(A\left[R:_{S} A\right]\right)^{\star_{1}} R[X]=R[X] .
$$

This shows that $A R[X]$ is $\star_{2}$-invertible.

Conversely, suppose that the $R[X]$-submodule $A R[X]$ is $\star_{2}$-invertible, and let $E=\left[R[X]:_{S[X]} A R[X]\right]$. Then $(A R[X] E)^{\star_{2}}=R[X]$. But by [12, Lemma 2.5(3)], we have $E=\left[R[X]:_{S[X]} A R[X]\right]=\left[R:_{S} A\right] R[X]$. Hence $R[X]=(A R[X] E)^{\star_{2}}=$ $\left(A R[X]\left[R:_{S} A\right] R[X]\right)^{\star_{2}}=\left(A\left[R:_{S} A\right] R[X]\right)^{\star_{2}}=\left(A\left[R:_{S} A\right]\right)^{\star_{1}} R[X]$. Therefore, $(A[R: S A])^{\star_{1}} R[X]=R[X]$. Hence $\left(A\left[R:_{S} A\right]\right)^{\star_{1}}=R$. This shows that $A$ is $\star_{1}$-invertible.

Lemma 3.10. Let $\star$ be a strict star operation on a ring extension $R \subseteq S$, and let $X$ be an indeterminate over $S$. Let $T$ be a multiplicatively closed subset of $N(\star)$. For each $R$-submodule $A$ of $S$, we have

$$
\left[R[X]_{[T]}:_{S[X]}(A R[X])_{[T]}\right]=\left[R[X]:_{[S[X]]} A R[X]\right]_{[T]}=\left(\left[R:_{S} A\right] R[X]\right)_{[T]} .
$$

Proof. The equality $\left[R[X]:_{S[X]} A R[X]\right]_{[T]}=\left(\left[R:_{S} A\right] R[X]\right)_{[T]}$ follows directly from $[12$, Lemma $2.5(3)]$. It remains to prove that $\left[R[X]_{[T]}:_{S[X]}(A R[X])_{[T]}\right]=$ $\left[R[X]:_{S[X]} A R[X]\right]_{[T]}$. Let $f \in\left[R[X]:_{S[X]} A R[X]\right]_{[T]}$. Then there exists $h_{1} \in T$ such that $h_{1} f \in\left[R[X]:_{S[X]} A R[X]\right]$. Hence $\left(h_{1} f\right) A R[X] \subseteq R[X]$. Let $g \in$ $(A R[X])_{[T]}$. Then there exists $h_{2} \in T$ such that $h_{2} g \in A R[X]$. So $\left(h_{1} h_{2}\right) f g=$ $\left(h_{1} f\right)\left(h_{2} g\right) \in R[X]$. Therefore, $f g \in R[X]_{[T]}$ since $h_{1} h_{2} \in T$. Hence $f(A R[X])_{[T]} \subseteq$ $R[X]_{[T]}$ since $g$ was arbitrary in $(A R[X])_{[T]}$. So $f \in\left[R[X]_{[T]}:_{S[X]}(A R[X])_{[T]}\right]$. This shows that $\left[R[X]:_{S[X]} A R[X]\right]_{[T]} \subseteq\left[R[X]_{[T]}:_{S[X]}(A R[X])_{[T]}\right]$.

Conversely, let $f \in\left[R[X]_{[T]}:_{S[X]}(A R[X])_{[T]}\right]$. Then $f\left(A R[X)_{[T]} \subseteq R[X]_{[T]}\right.$. It follows that $f A \subseteq R[X]_{[T]}$ since $A \subseteq(A R[X])_{[T]}$. Let $a \in A$. Then af $\in R[X]_{[T]}$. So there exists $h \in T$ such that $h(a f) \in R[X]$. Hence $c_{R}(h a f) \subseteq R$. It follows that $c_{R}(h a f) c_{R}(h)^{\ell} \subseteq R$ for each positive integer $\ell$ since $h \in R[X]$. But by the DedekindMertens formula ([8, Theorem 1.1,p. 126]), we have $c_{R}(h)^{\ell_{0}+1} c_{R}($ af $)=c_{R}($ haf $) c_{R}(h)^{\ell_{0}} \subseteq$ $R$ for some positive integer $\ell_{0}$. Hence

$$
c_{R}(h)^{\ell_{0}+1} c_{R}(a f)^{\star} \subseteq\left(c_{R}(h)^{\ell_{0+1}} c_{R}(a f)\right)^{\star}=\left(c_{R}(h a f) c_{R}(h)^{\ell_{0}}\right)^{\star} \subseteq R^{\star}=R .
$$

It follows from [8, Proposition 4.1 (a), p. 146] that $\left(\left(c_{R}(h)^{\ell_{0}+1}\right)^{\star} c_{R}(a f)^{\star}\right)^{\star} \subseteq R^{\star}=$ $R$. But by Remark 1.2 we have $\left(c_{R}(h)^{\ell_{0}+1}\right)^{\star}=R^{\star}=R$ since $c_{R}(h)^{\star}=R$. It follows that $\left(c_{R}(a f)^{\star} R\right)^{\star} \subseteq R$. Thus $c_{R}(a f)^{\star} \subseteq R$. This shows that af $\in R[X]$. So 
$f A \subseteq R[X]$ since $a$ was arbitrary chosen in $A$. Hence $f(A R[X]) \subseteq R[X]$. Thus $f \in\left[R[X]:_{S[X]} A R[X]\right]$. Therefore $\left[R[X]_{[T]}:_{S[X]}(A R[X])_{[T]}\right] \subseteq\left[R[X]:_{S[X]} A R[X]\right]$. Hence $\left[R[X]_{[T]}:_{S[X]}(A R[X])_{[T]}\right]=\left[R[X]:_{S[X]} A R[X]\right]$.

Lemma 3.11. ([12, Lemma 3.9]) Let $R \subseteq S$ be a ring extension, and $Q$ be a maximal ideal of $R[X]$ satisfying $\left(R[X]:_{S[X]} Q\right) \neq R[X]$. Then $Q \cap R=0$ or $(Q \cap R)[X]=Q$.

The next result is a generalization of [6, Corollary 2.5] which states that in a domain $R$, a nonzero ideal $I$ is $t$-invertible if and only if $I[X]_{N_{v}}$ is invertible.

Theorem 3.12. Let $R \subseteq S$ be a ring extension, and let $X$ be an indeterminate over $S$. A nonzero ideal $I$ of $R$ is $S$-invertible if and only if $I R[X]_{[N(d)]}$ is an $S[X]$-invertible ideal of $R[X]_{[N(d)]}$.

Proof. Let $I$ be a nonzero ideal of $R$. Suppose that $I$ is $S$-invertible. Then $I I^{-1}=R$. We show that $I R[X]_{[N(d)]} I^{-1} R[X]_{[N(d)]}=R[X]_{[N(d)]}$. By contradiction, suppose that $I R[X]_{[N(d)]} I^{-1} R[X]_{[N(d)]}$ is a proper ideal of $R[X]_{[N(d)]}$. Then, $I R[X]_{[N(d)]} I^{-1} R[X]_{[N(d)]} \subseteq J$ for some maximal ideal $J$ of $R[X]_{[N(d)]}$. So, $I I^{-1}[X] \subseteq I[X] I^{-1}[X] \subseteq I R[X]_{[N(d)]} I^{-1} R[X]_{[N(d)]} \subseteq J$. Hence $I I^{-1}[X] \subseteq$ $Q=J \cap R[X]$. Furthermore, $Q \cap R \neq 0$ since $R=I I^{-1} \subseteq I I^{-1}[X] \subseteq Q$. Thus $Q \cap R \neq 0$. Let $Q^{\prime}$ be a maximal ideal of $R[X]$ such that $Q \subseteq Q^{\prime}$. Then $\mathfrak{m}=Q^{\prime} \cap R \neq 0$ since $Q \cap R \neq 0$. It follows from the previous lemma that $\mathfrak{m}$ is a maximal ideal of $R$ satisfying $\mathfrak{m}[X]=Q^{\prime}$. We show that $Q^{\prime} \cap N(d)=\varnothing$. By contradiction, $Q^{\prime} \cap N(d)=\mathfrak{m}[X] \cap N(d) \neq \varnothing$. Then there exists $f=\sum_{i=0}^{\ell} a_{i} X^{i} \in \mathfrak{m}[X]$ with $a_{0} \ldots a_{\ell} \in \mathfrak{m}$ such that $R=c_{R}(f)$. It follows that $R=\left(a_{0}, \ldots, a_{\ell}\right) R \subseteq$ $\mathfrak{m}$. This is a contradiction since $\mathfrak{m}$ is a proper ideal of $R$. This shows that $Q^{\prime} \cap N(d)=\mathfrak{m}[X] \cap N(d)=\varnothing$. Therefore, by Proposition 3.7(2), we have $Q_{[N(d)]}=\mathfrak{m}[X]_{[N(d)]}$ is a maximal ideal of $R[X]_{[N(d)]}$. Furthermore, by Remark $2.2(1), J \subseteq J_{[N(d)]}=(J \cap R[X])_{[N(d)]}=Q_{[N]}=\mathfrak{m}[X]_{[N(d)]}$. It follows from the maximality of $J$ that $J=\mathfrak{m}[X]_{[N(d)]}$.

Conversely, suppose that $I R[X]_{[N(d)]}$ is an $S[X]$-invertible ideal of $R[X]_{[N(d)]}$. Then $\operatorname{IR}[X]_{[N(d)]}\left(\operatorname{IR}[X]_{[N(d)]}\right)^{-1}=R[X]_{[N(d)]}$. Therefore, by Lemma 3.10, we have $I R[X]_{[N(d)]}\left(I^{-1} R[X]\right)_{[N(d)]}=R[X]_{[N(d)]}$. Furthermore,

$$
I R[X]_{[N(d)]}\left(I^{-1} R[X]\right)_{[N(d)]} \subseteq\left(I R[X] I^{-1} R[X]\right)_{[N(d)]} .
$$

Thus $\left(I I^{-1} R[X]\right)_{[N(d)]}=\left(I R[X] I^{-1} R[X]\right)_{[N(d)]}=R[X]_{[N(d)]}$. By contradiction, suppose that $I I^{-1}$ is contained in a maximal ideal $\mathfrak{m}$ of $R$. Then $\left(I I^{-1} R[X]\right)_{[N(d)]} \subseteq$ $\mathfrak{m} R[X]_{[N(d)]}$, and we have $R[X]_{[N(d)]} \subseteq \mathfrak{m} R[X]_{[N(d)]}$. This is a contradiction since 
by Corollary 3.8, $\mathfrak{m} R[X]_{[N(d)]}$ is a maximal ideal of $R[X]_{[N(d)]}$. This shows that $I I^{-1}$ is not contained in any maximal ideal of $R$. It follows from Lemma 1.3 that $I I^{-1}=R$.

Theorem 3.13. Let $R \subseteq S$ be a ring extension, and let $X$ be an indeterminate over $S$ such that $R[X] \subseteq S[X]$ is integrally closed. Let $\mathfrak{m}$ be a maximal ideal of $R$. Then $R_{[\mathfrak{m}]} \subseteq S$ is a Manis extension if and only if $R[X]_{[\mathfrak{m} R[X]]} \subseteq S[X]$ is a Manis extension.

Proof. Suppose that the extension $R_{[\mathfrak{m}]} \subseteq S$ is Manis. Then by Remark 1.4, the extension $R_{[\mathfrak{m}]}[X] \subseteq S[X]$ is also Manis. But by Lemma 2.6(1), we have $R_{[\mathfrak{m}]}[X]=R[X]_{[\mathfrak{m} R[X]]}$. Thus the extension $R[X]_{[\mathfrak{m} R[X]]} \subseteq S[X]$ is Manis.

Conversely, suppose that the extension $R[X]_{[\mathfrak{m} R[X]]} \subseteq S[X]$ is Manis. Let $\alpha$ be an element of $S$. Then $\alpha \in S[X]$. Therefore, by [7, Theorem 2.12, p. 29], there exists a polynomial $F(Y) \in(S[X])[Y] \backslash(\mathfrak{m} R[X])[Y]$ such that $F(\alpha)=0$, where $Y$ is an indeterminate over $S[X]$. Write $F(Y)=\sum_{i=0}^{n} f_{i}(X) Y^{i}$. Then $\sum_{i=0}^{n} f_{i}(X) \alpha^{i}=0$. Write $f_{i}(X)=\sum_{j=0}^{\ell} a_{i j} X^{j}$ for a fixed positive integer $\ell$, with possibly some of the $a_{i j}$ 's equal zero. Then $\sum_{i=0}^{n}\left(\sum_{j=0}^{\ell} a_{i j} X^{j}\right) \alpha^{i}=\sum_{j=0}^{\ell}\left(\sum_{i=0}^{n} a_{i j} \alpha^{i}\right) X^{j}=0$. It follows that

$$
\sum_{i=0}^{n} a_{i j} \alpha^{i}=0 \text { for each } 1 \leqslant j \leqslant \ell
$$

Since $F(Y) \notin(\mathfrak{m} R[X])[Y]$, there exists $1 \leqslant k \leqslant n$ such that $f_{k}(X) \notin \mathfrak{m} R[X]$. Thus $f_{k}(X) \in S[X] \backslash \mathfrak{m} R[X]$. So there exists $1 \leqslant j_{0} \leqslant \ell$ such that $a_{k j_{0}} \notin \mathfrak{m}$. Let $g(X)=$ $\sum_{i=0}^{n} a_{i j_{0}} X^{i}$. Then by $(\Theta)$, we have $g(\alpha)=0$. Furthermore, $g(X) \in S[X] \backslash \mathfrak{m}[X]$ since $a_{k j_{0}} \notin \mathfrak{m}$. By [7, Theorem 2.12, p. 29], the extension $R_{[\mathfrak{m}]} \subseteq S$ is Manis.

Corollary 3.14. Let $R \subseteq S$ be a ring extension. If the extension $R[X]_{[N(d)]} \subseteq$ $S[X]$ is Prüfer, then the extension $R \subseteq S$ is Prüfer.

Proof. Suppose that $R[X]_{[N(d)]} \subseteq S[X]$ is a Prüfer extension, and let $\mathfrak{m}$ be a maximal ideal of $R$. By Proposition $3.7(2), \mathfrak{m}[X]_{[N(d)]}$ is a maximal ideal of $R[X]_{[N(d)]}$. It follows from the hypothesis that $\left(R[X]_{[N(d)]}\right)_{\left[\mathfrak{m}[X]_{[N(d)]}\right]} \subseteq S[X]$ is a Manis extension. But by Lemma 2.6(2), we have $\left(R[X]_{[N(d)]}\right)_{\left[\mathfrak{m}[X]_{[N(d)]}\right]}=R[X]_{[\mathfrak{m}[X]]}$. Furthermore, by Lemma 2.6(1) we have $R[X]_{[\mathfrak{m}[X]]}=R_{[\mathfrak{m}]}[X]$. This shows that $R_{[\mathfrak{m}]}[X] \subseteq S[X]$ is a Manis extension. It follows from Proposition 3.13 that the extension $R_{[\mathfrak{m}]} \subseteq S$ is Manis. This shows that $R \subseteq S$ is a Prüfer.

Before proving the next result, we recall the definition of a Prüfer star multiplication extension, and the definition of a weak Prüfer star multiplication extension. 
These notions are introduced and studied in [10]. Let $R \subseteq S$ be a ring extension, and let $\star: \mathcal{J}(R, S) \longrightarrow \mathcal{J}(R, S)$ be a star operation. The ring extension $R \subseteq S$ is called weak Prüfer star-multiplication extension (weak $P \star M E$ ) if the pair $\left(R_{[\mathfrak{m}]}, \mathfrak{m}_{[\mathfrak{m}]}\right)$ is Manis in $S$ for every $S$-regular $\star$-maximal ideal $\mathfrak{m}$ of $R$. When the pair $\left(R_{[\mathfrak{m}]}, \mathfrak{m}_{[\mathfrak{m}]}\right)$ is Manis in $S$ for every $\star$-maximal ideal $\mathfrak{m}$ of $R$, the extension $R \subseteq S$ is called Prüfer star-multiplication extension ( $P \star M E)$ [10, Definition 3.1].

Theorem 3.15. Consider the following pullback diagram of type $\square$ with $\operatorname{ker} \Psi \subseteq$ $\operatorname{Jac}(R)$.

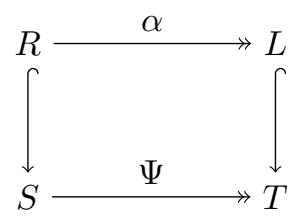

Let $\star_{1}$ be a star operation on $R \subseteq S$, and let $\star_{2}$ be a star operation on the extension $L \subseteq T$ such that $\Psi\left(A^{\star_{1}}\right)=\Psi(A)^{\star_{2}}$ for each $A \in \mathcal{J}(R, S)$. Then $R \subseteq S$ is weak $P \star_{1} M E$ if and only if $L \subseteq T$ is weak $P \star_{2} M E$.

Proof. Suppose that $R \subseteq S$ is a weak $\mathrm{P} \star_{1}$ ME. Let $\mathfrak{n}$ be a $T$-regular $\star_{2}$-maximal ideal of $L$, and let $\mathfrak{m}=\Psi^{-1}(\mathfrak{n})$. Then by Lemma 2.4(1), $\mathfrak{m}$ is an ideal of $R$ satisfying $\mathfrak{m} S=S$. But $\Psi(\mathfrak{m})=\mathfrak{n}=\mathfrak{n}^{\star_{2}}=\Psi(\mathfrak{m})^{\star_{2}}=\Psi\left(\mathfrak{m}^{\star_{1}}\right)$. It follows from Lemma 2.4(3) that $\mathfrak{m}=\mathfrak{m}^{\star_{1}}$. This shows that $\mathfrak{m}$ is a $\star_{1}$-ideal. Let $I$ be a $\star_{1}$-ideal of $R$ such that $\mathfrak{m} \subseteq I$. Then $I S=S$ since $\mathfrak{m} S=S$. Furthermore, $\Psi(I)$ is a $\star_{2}$-ideal of $L$ since $\Psi(I)^{\star_{2}}=\Psi\left(I^{\star_{1}}\right)=\Psi(I)$. Hence $\Psi(I)$ is a $\star_{2}$-maximal ideal of $L$. But $\mathfrak{n}=\Psi(\mathfrak{m}) \subseteq \Psi(I)$. It follows from the maximality of $\mathfrak{n}$ that $\Psi(\mathfrak{m})=\Psi(I)$ or $\Psi(\mathfrak{m})=\Psi(R)$. Therefore, by Lemma 2.4(3), we have $\mathfrak{m}=I$ or $\mathfrak{m}=R$. This shows that $\mathfrak{m}$ is an $S$-regular $\star_{1}$-maximal ideal of $R$. It follows from the hypothesis that $\left(R_{[\mathfrak{m}]}, \mathfrak{m}_{[\mathfrak{m}]}\right)$ is a Manis pair of $S$. Let $v$ be an element of $T$. There exists $u \in S$ such that $v=\Psi(u)$. Then by [7, Theorem 2.12, p. 29], there exists a polynomial $F(X)=\sum_{i=1}^{\ell} a_{i} X^{i} \in R[X] \backslash \mathfrak{m}[X]$ such that $F(u)=0$. Let $G(X)=\sum_{i=1}^{\ell} \Psi\left(a_{i}\right) X^{i}$. Then $G(v)=\sum_{i=1}^{\ell} \Psi\left(a_{i}\right) v^{i}=\sum_{i=1}^{\ell} \Psi\left(a_{i}\right) \Psi(u)^{i}=\Psi(F(u))=0$. Furthermore, since $F(X) \in R[X] \backslash \mathfrak{m}[X]$, there exists $j, 1 \leqslant j \leqslant \ell$ such that $a_{j} \in R \backslash \mathfrak{m}$. We show that $\Psi\left(a_{j}\right) \notin \mathfrak{n}$. By contradiction, suppose that $\Psi\left(a_{j}\right) \in \mathfrak{n}$. Then there exists $a \in \mathfrak{m}$ such that $\Psi\left(a_{j}\right)=\Psi(a)$. Hence $a_{j}-a \in \operatorname{ker} \Psi$. But by Lemma $2.4(1)$, $\operatorname{ker} \Psi \subseteq \mathfrak{m}$. It follows that $a_{j} \in \mathfrak{m}$. This is a contradiction since $a_{j} \in R \backslash \mathfrak{m}$. We have shown that $G(X) \in L[X] \backslash \mathfrak{n}[X]$ and $G(v)=0$. It follows from [7, Theorem 2.12, p. 29] that 
$\left(L_{[\mathfrak{n}]}, \mathfrak{n}_{[\mathfrak{n}]}\right)$ is a Manis pair in $T$, since $v$ was arbitrary chosen in $T$. This shows that the extension $L \subseteq T$ is a weak $\mathrm{P} \star_{2} \mathrm{ME}$.

Conversely, suppose that the extension $L \subseteq T$ is a weak $\mathrm{P} \star_{2} \mathrm{ME}$. Let $\mathfrak{m}$ be an $S$-regular $\star_{1}$-maximal ideal of $R$, and let $\mathfrak{n}=\Psi(\mathfrak{m})$. Since $\mathfrak{m} S=S$, there exist $r_{1}, \ldots, r_{k} \in \mathfrak{m}$ and $s_{1}, \ldots, s_{k} \in S$ such that $\sum_{i=1}^{k} r_{i} s_{i}=1$. Hence $\sum_{i=1}^{k} \Psi\left(r_{i}\right) \Psi\left(s_{i}\right)=$ $\Psi(1)=1$. This shows that $\mathfrak{n} S=S$ since $\Psi\left(r_{i}\right) \in \mathfrak{n}$ for $1 \leqslant i \leqslant k$. Also, $\mathfrak{n}^{\star_{2}}=$ $\Psi(\mathfrak{m})^{\star_{2}}=\Psi\left(\mathfrak{m}^{\star_{1}}\right)=\Psi(\mathfrak{m})=\mathfrak{n}$. Thus $\mathfrak{n}$ is a $\star_{2}$-ideal of $L$. Suppose that $J$ is a $\star_{2}$-ideal of $L$ such that $\mathfrak{n} \subseteq J$. Then $J S=S$ since $\mathfrak{n} S=S$. Let $I=\Psi^{-1}(J)$. Then by Lemma $2.4(2), I S=S$. Furthermore, $\Psi(\mathfrak{m}) \subseteq \Psi(I)$. It follows from $[9$, Remark $2.5]$ that $\mathfrak{m} \subseteq I$. Therefore, by the maximality of $\mathfrak{m}$, we have $\mathfrak{m}=I$ or $I=R$. Hence $\mathfrak{n}=J$ or $J=L$. This shows that $\mathfrak{n}$ is a $T$-regular $\star_{2}$-maximal ideal of $L$. It follows from the hypothesis that $\left(L_{[\mathfrak{n}]}, \mathfrak{n}_{[\mathfrak{n}]}\right)$ is a Manis pair of $T$. Let $u$ be an element of $S$. Then $v=\Psi(u)$ is an element of $T$. By [7, Theorem 2.12, p. 29], there exists a polynomial $G(X)=\sum_{i=0}^{\ell} b_{i} X^{i} \in L[X] \backslash \mathfrak{n}[X]$ such that $G(v)=0$. Since $L=\Psi(R)$, there exist $a_{0}, \ldots, a_{\ell} \in R$ such that $b_{i}=\Psi\left(a_{i}\right)$ for $1 \leqslant i \leqslant \ell$. Let $F(X)=\sum_{i=0}^{\ell} a_{i} X^{i}$. Then $\Psi(F(u))=G(v)=0$. Hence $F(u) \in \operatorname{ker} \Psi \subseteq R$. Therefore $F(u)=r$ for some $r \in R$. Let $H(X)=F(X)-r \in R[X]$. Then $H(u)=0$. We show that $H(X) \notin \mathfrak{m}[X]$. Since $G(X) \notin \mathfrak{n}[X]$, there exists $j_{0}$ such that $b_{j_{0}} \notin \mathfrak{n}, 1 \leqslant j_{0} \leqslant \ell$. By contradiction, suppose that $a_{j_{0}} \in \mathfrak{m}$. Then $b_{j_{0}}=\Psi\left(a_{j_{0}}\right) \in \Psi(\mathfrak{m})=\mathfrak{n}$. This is a contradiction. Hence $a_{j_{0}} \notin \mathfrak{m}$. This shows that $F(X) \in R[X] \backslash \mathfrak{m}[X]$. It follows that $H(X) \in R[X] \backslash \mathfrak{m}[X]$. We have shown that $H(X) \in R[X] \backslash \mathfrak{m}[X]$ and $H(u)=0$. It follows from [7, Theorem 2.12, p. 29] that $\left(R_{[\mathfrak{m}]}, \mathfrak{m}_{[\mathfrak{m}]}\right)$ is a Manis pair in $S$. Thus the extension $R \subseteq S$ is a weak $\mathrm{P} \star_{1} \mathrm{ME}$.

Remark 3.16. The notion of $\star$-multiplication domain is introduced and studied [5]. A domain $D$ is called $\star$-multiplication domain if for each nonzero finitely generated ideal $I$ of $D$, there exists a finitely generated fractional ideal $J$ of $D$ satisfying $(I J)^{\star}=D$, where $\star$ is a star operation on $D$. The name Prüfer $v$-multiplication domain (PVMD) has been used for $v$-multiplication domain, where the $v$-operation is defined by $I_{v}=\left(I^{-1}\right)^{-1}$; see for example [4]. Several equivalent conditions to the notion of $\star$-multiplication domains are given in $[5$,$] . In particular, it is shown that$ a domain $D$ is a $\star$-multiplication domain if and only if $D_{M}$ is a valuation domain for each ideal $M$ maximal in the set of $\star$-ideals, where $\star$ is a star operation of finite type on $D$ [5, Theorem 1.1]. In this paper, the definition of Prüfer $\star$-multiplication extension involves Manis extensions. This yields the following open question: Is it possible to characterize Prüfer $\star$-multiplication extension with $\star$-invertibility? In 
[10, Proposition 2.4], it is shown that for a ring extension $R \subseteq S$ with $Q(R) \subseteq S$ (where $Q(R)$ is the total ring of fractions of $R$ ), and $\star: \mathcal{J}(R, S) \longrightarrow \mathcal{J}(R, S)$ a star operation of finite type, if $R \subseteq S$ is a (weak) $\mathrm{P} \star \mathrm{ME}$, then each finitely generated $S$-regular $R$-submodule $A$ of $S$ is $\star$-invertible.

Acknowledgement. We are thankful to the referee for the valuable comments and suggestions that helped to improve the quality of this work.

\section{References}

[1] M. Fontana and M. Zafrullah, On v-domains: a survey. Commutative algebraNoetherian and non-Noetherian perspectives, 145-179, Springer, New York, 2011.

[2] S. Gabelli and E. Houston, Ideal theory in pullbacks, In: Chapman S. T., Glaz S., eds. Non-Noetherian Commutative Ring Theory, Math. Appl., vol. 520, Kluwer Academic Publishers, Dordrecht (2000), 199-227.

[3] R. Gilmer, Multiplicative Ideal Theory, Corrected reprint of the 1972 edition, Queen's Papers in Pure and Applied Mathematics, 90, Queen's University, Kingston, ON, 1992.

[4] M. Griffin, Some results on v-multiplication rings, Canadian J. Math., 19 (1967), 710-722.

[5] E. G. Houston, S. B. Malik and J. Mott, Characterizations of $\star-$ multiplication domains, Canadian Math. Bull., 27(1) (1984), 48-52.

[6] B. G. Kang, Prüfer v-multiplicative domains and the ring $R[X]_{N_{v}}$, J. Algebra, 123 (1989), 151-170.

[7] M. Knebusch and D. Zhang, Manis Valuations and Prüfer Extensions. I, Lecture Notes in Mathematics, 1791, Springer-Verlag, Berlin, 2002.

[8] M. Knebusch and T. Kaiser, Manis Valuations and Prüfer Extensions II, Lectures Notes in Mathematics, 2103, Springer, Cham, 2014.

[9] L. Paudel and S. Tchamna, Pullback diagrams and Kronecker function rings, Rocky Mountain J. Math., 49(7)(2019), 2267-2279.

[10] L. Paudel and S. Tchamna, A study of linked star operations, to appear in Bull. Korean Math. Soc.

[11] S. Tchamna, Multiplicative canonical ideals of ring extension, J. Algebra Appl., 16(4) (2017), 1750069 (15 pp).

[12] S. Tchamna, On ring extensions satisfying the star-hash property, Comm. Algebra, 48(5) (2020), 2081-2091. 


\section{Lokendra Paudel}

Department of Mathematics

University of South Carolina

Salkehatchie, SC 29810, USA

e-mail: lpaudel@mailbox.sc.edu

Simplice Tchamna (Corresponding Author)

Department of Mathematics

Georgia College \& State University

Campus Box 017, Millegeville, GA 31061, USA

e-mail: simplice.tchamna@gcsu.edu 